\title{
Genome-wide interaction analysis identified low-frequency variants with sex disparity in lung cancer risk
}

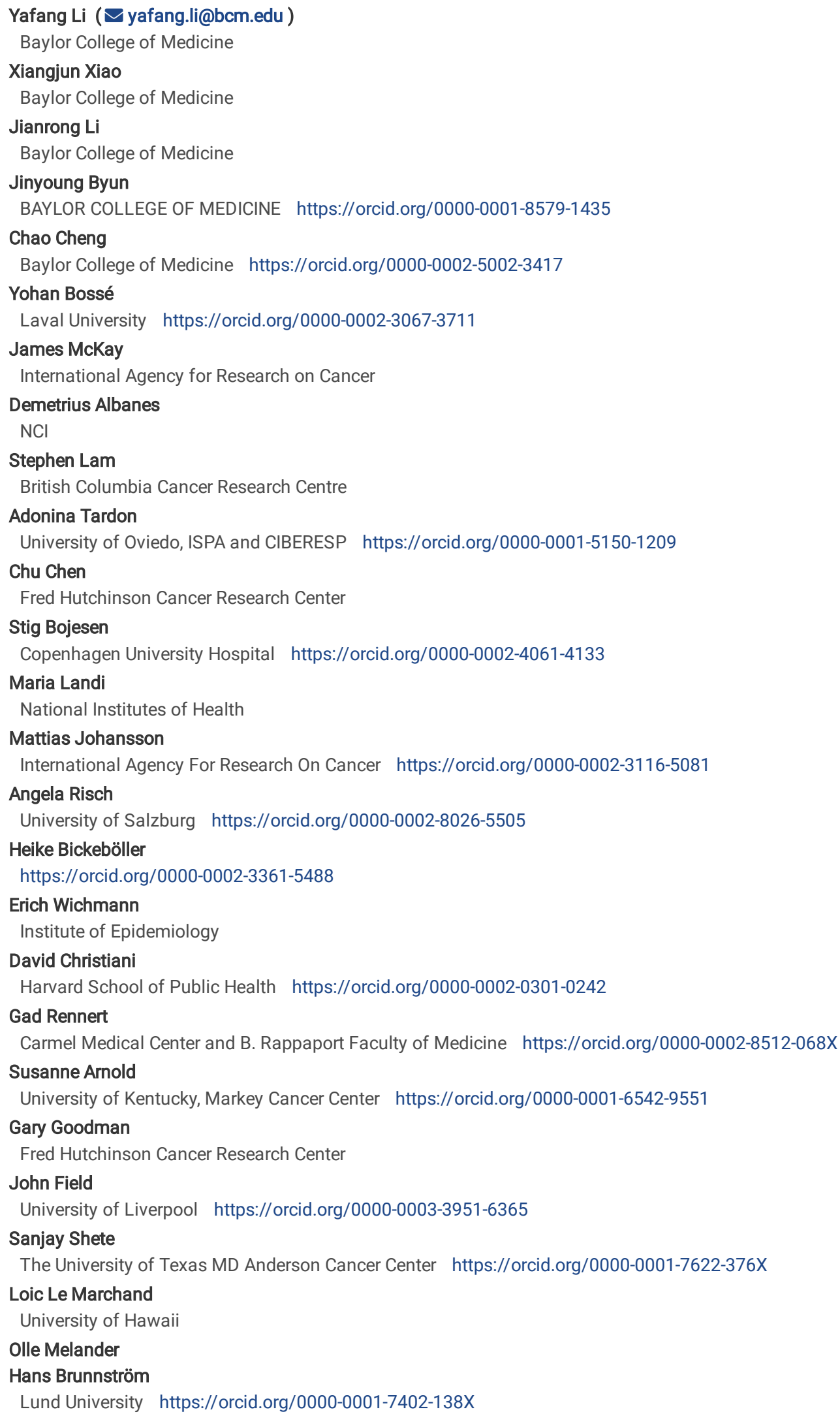




\section{Geoffrey Liu}

University Health Network, Ontario Cancer Institute/Princess Margaret Hospital

\section{Rayjean Hung}

Lunenfeld-Tanenbaum Research Institute https://orcid.org/0000-0002-4486-7496

Angeline Andrew

Dartmouth Medical School

Lambertus Kiemeney

Radboudumc https://orcid.org/0000-0002-2368-1326

\section{Hongbing Shen}

Nanjing Medical University

Ryan Sun

University of Texas MD Anderson Cancer Center

Shanbeh Zienolddiny

National Instutute of Occupational Health

Kjell Grankvist

Umeå University https://orcid.org/0000-0003-4289-2097

Mikael Johansson

Department of Radiation Sciences, Umeå University 9 https://orcid.org/0000-0003-4182-8923

Neil Caporaso

Division of Cancer Epidemiology and Genetics, National Cancer Institute, National Institutes of Health

\section{Dawn Teare}

University of Newcastle https://orcid.org/0000-0003-3994-0051

Yun-Chul Hong

Seoul National University College of Medicine,

\section{Michael Davies}

University of Liverpool https://orcid.org/0000-0002-7609-4977

\section{Philip Lazarus}

Department of Pharmaceutical Sciences, College of Pharmacy, Washington State University

\section{Matthew Schabath}

Moffitt Cancer Center https://orcid.org/0000-0003-3241-3216

Melinda Aldrich

Vanderbilt University Medical Center

Ann Schwartz

Karmanos Cancer Institute Wayne State University School of Medicine

Ivan Gorlov

Baylor College of Medicine

Kristen Purrington

Karmanos Cancer Institute

Ping Yang

Mayo Clinic

Younghun Han

Baylor College of Medicine

Joan Bailey-Wilson

National Human Genome Research Institute, National Institutes of Health

Yanhong Liu

Baylor College of Medicine

Susan Pinney

University of Cincinnati College of Medicine

Diptasri Mandal

Louisiana State University Health Sciences Center

James Willey

University of Teledo Medical Center

Colette Gaba

University of Toledo

Paul Brennan

International Agency for Research on Cancer (IARC/WHO) https://orcid.org/0000-0002-0518-8714

Christopher Amos

Baylor College of Medicine 
Article

Keywords: lung cancer risk, sex disparity, genetics, molecular genetics

Posted Date: July 13th, 2021

DOI: https://doi.org/10.21203/rs.3.rs-647012/v1

License: (c) (i) This work is licensed under a Creative Commons Attribution 4.0 International License. Read Full License

Version of Record: A version of this preprint was published at Human Molecular Genetics on January 1st, 2022. See the published version at https://doi.org/10.1093/hmg/ddac030. 


\section{Abstract}

There is growing evidence showing that sex disparity in lung cancer risk cannot be fully explained by sex differences in smoking behavior, implying existence of genetic and molecular basis for sex disparity in lung cancer development. However, the information about sex dimorphism in lung cancer risk is quite limited despite the great success in lung cancer association studies. By adopting a stringent two-stage analysis strategy, we performed a genome-wide genesex interaction analysis using genotype from a lung cancer cohort including 47,000 individuals with European ancestry. Three low-frequency variants (minor allele frequency < 0.05), rs17662871 (OR=0.71, p=4.29x10-8); rs79942605 (OR=2.17, p=2.81x10-8); and rs208908 (OR=0.70, $p=4.54 \times 10-8)$, were identified with different risk effect of lung cancer between men and women. Further eQTL and functional annotation analysis suggested rs208908 affect lung cancer risk through differential regulation of CXADR (Coxsackie Virus And Adenovirus Receptor) gene expression in lung tissues between men and women. Our study is one of the first studies to provide novel insights about the genetic and molecular basis for sex disparity in lung cancer development.

\section{Introduction}

Lung cancer is the leading cause of cancer death for both men and women worldwide with a complex genetic and molecular mechanism. Differences by sex in lung cancer incidence and mortality have been reported ${ }^{1-2}$. Historically, the sex difference in lung cancer risk development was explained by sex-differences in smoking behavior as women were less likely to smoke cigarettes, initiated smoking at older ages, and smoked fewer cigarettes per day ${ }^{3-5}$. However, as smoking behavior has become increasingly similar between men and women, there is growing evidence that sex differences in lung cancer risk cannot be fully explained by differences in smoking behavior, implying sex-based variations in the genetic and molecular basis for lung cancer ${ }^{6}$. However, the information for sex differences in lung cancer risk remains poorly understood despite the extensive efforts spent in lung cancer research and great success in identifying genetic factors through lung cancer association studies.

Genome-wide association studies (GWAS) have been used to identify sexual dimorphism in genetic susceptibility to lung cancer. Sex-specific GWAS identified $V T I 1 A, A C V R 1 B$ and FOXP4-AS1 genes in women that influence lung cancer development ${ }^{7-8}$. However, prior female sex-specific GWAS could not test for statistical significance in males or for gene-sex interactions. Gene-sex interaction analysis, on the other hand, will evaluate the information from both male and female group systematically and can identify the variants with significant difference between men and women, although those variants may not achieve genome-wide significance in stratified analysis. But genome-wide genetic interaction (GWGI) studies still remain challenging as most genome-wide association studies were designed for main effect association analysis and have had limited power for interaction analysis. A two-step analysis has been proposed and utilized in GWGI: step 1, a case-only analysis to test the association between SNPs and environmental/or biological factors; step 2, candidate SNPs from step 1 are further submitted to standard case-control logistic interaction analysis ${ }^{9}$. This two-step study design benefits from robustness from case-only analysis and stringency from case-control analysis and has been applied in a number of GWGI studies ${ }^{10}$.

Functional inference of genetic variants is important for gaining insights about the molecular mechanism of the disease and clinical application of GWAS findings. Over $90 \%$ of GWAS variants are located within non-coding regions and they may affect disease risk through regulating expression of nearby genes $^{11-12}$. Expression quantitative trait loci (eQTL) analysis, an allelic association analysis with gene expression, provides a straightforward method to identify susceptibility genes associated with the GWAS hits and it has been widely used in GWAS to investigate the regulatory effect of variants ${ }^{13-15}$. However, there are few or no reports about the application of eQTL in gene-sex interaction analysis in human diseases because of the limited studies in sexual dimorphism in disease risk. Another important approach for assessment of genetic variants is functional annotation analysis. Researchers developed various tools to infer the functional roles for the variants such as CADD (Combined Annotation Dependent Depletion) and RegulomeDB ${ }^{16-18}$. These tools provide insightful information about the functional inference and are very helpful for fine mapping and pinning down the true causal allele.

In this report, we performed a genome-wide gene-sex interaction analysis using genotype from a large lung cancer cohort including $~ 47,000$ individuals with European ancestry. Adopting the two-step analysis strategy, we identified novel variants with different risk effects between men and women that were missed by main-effect association analysis. eQTL and functional annotation analysis provided further information about the functional role for the identified genetic variants and supplied multiple lines of evidence for the sexual disparity in lung cancer development. Our study is one of the first studies for sexual difference in risk of cancer development between men and women. It is also the largest scanning for gene-sex interaction in lung cancer and explored the genetic and molecular basis for sexual differences in risk of this deadly disease.

\section{Material And Methods}

\section{Materials}

The imputed genotypes (reference panel: HRC r1.1) from 46,783 individuals from European population, with lung cancer phenotype, smoking (ever- vs neversmokers) and sex information, in the INTEGRAL (Integrative Analysis of Lung Cancer Etiology and Risk)-ILCCO (International Lung Cancer Consortium) lung cancer consortium were analyzed in this study ${ }^{19}$. The subjects came from 9 independent studies: the OncoArray Consortium Lung Study (OncoArray), including 16,845 lung cancer cases and 13,057 controls, was used as the discovery dataset ${ }^{20-21}$. Individuals from another 8 smaller independent studies: Affymetrix Axiom Array Study (AFFY), the Genetic Epidemiology of Lung Cancer (GELCC) Consortium, the Environment and Genetics in Lung cancer Etiology study (EAGLE), the International Agency for Research on Cancer (IARC), MD Anderson Cancer Center Study (MDACC), NCI Lung Cancer and Smoking Phenotypes in African-American Cases and Controls ( $\mathrm{NCl}$ ), the Prostate, Lung, Colorectal and Ovarian Cancer Screening Trial (PLCO), and Samuel Lunenfeld Research Institute Study (SLRI), were combined together and used as replication dataset including 7,378 lung cancer cases and 9,503 controls (Table 1, Supplementary Table S1) ${ }^{22-25}$. There were 12,084 women and 17,818 men in discovery dataset; and 6,366 women and 10,515 men in replication dataset. $60.4 \%$ of the lung cancer patients were male in the discovery study compared with $64.0 \%$ in replication study. $81.6 \%$ of individuals in both discovery and 
validation studies were ever-smokers. Stringent quality controls were applied on SNPs and SNPs selected for the analysis had to qualify by two criteria: 1 , had imputation information score $>=0.7$ in discovery data; 2 , had information score $>0.2$ in each of the eight studies and sample-size weighted information score $>=0.7$ in replication data. About $\sim 20,000,000$, out of 39,000,000 imputed SNPs, with information score > = 0.7 were analyzed in discovery study; and $~$ $10,000,000$ SNPs were analyzed in replication study (Fig. 1). 193,050 markers, common to the 9 studies and with linkage disequilibrium $r^{2}$ value less than or equal to 0.5 , were selected to calculate principal components in PLINK. Detailed information about data collection, genotype imputation and quality control procedures can be found from our earlier publication ${ }^{19}$.

Table 1

Characteristics of participants that were studied during the Discovery and Replication phases.

\begin{tabular}{|llllll|}
\hline & \multicolumn{1}{c|}{ Discovery ${ }^{1}$} & \multicolumn{3}{c|}{ Replication $^{2}$} \\
\hline & Male (\%) & Female (\%) & Total & Male (\%) & Female (\%) \\
\hline Controls & $7646(58.6)$ & $5411(41.4)$ & 13057 & $5796(61.0)$ & $3707(39.0)$ \\
\hline Cases & $10172(60.4)$ & $6673(39.6)$ & 16845 & $4719(64.0)$ & $2659(36.0)$ \\
\hline Ever-smokers & $15584(63.9)$ & $8813(36.1)$ & 24397 & $9230(67.0)$ & $4553(33.0)$ \\
\hline Never-smokers & $2234(40.6)$ & $3271(59.4)$ & 5505 & $1285(41.5)$ & $1813(58.5)$ \\
\hline
\end{tabular}

Samples with European ancestry in INTEGRAL-ILCCO consortium were analyzed in the study. Number of overall lung cancer cases was provided in the table. 1, genotype data from Oncoarray study was used in Discovery study; 2 , genotype from the other 8 studies were used in replication study.

\section{Statistical Methods For Gwgi}

Following the two-step analysis strategy, case-only analysis was first performed between SNP dosage (additive model) and sex (male and female were coded as 1 and 2, respectively) phenotype using lung cancer patients from discovery study $(N=16,845)$ and replication study ( $N=7,378)($ Case-only model, $S$ denotes smoking status). Fixed-effect meta-analysis was conducted to combine information from both studies. Variants selected for further case-control analysis had to satisfy all three criteria: 1 , with case-only joint $p$-value $<5 \times 10^{-8} ; 2$, case-only $p<0.1$ in replication study; and 3 , same direction of effect in both studies. All the samples in discovery and replication study including 24,223 lung cancer patients and 22,560 controls were applied in case-control analysis (Case-control model, D denotes disease status). The SNPs with case-control gene-sex interaction $p$-value $<0.05$ and same direction of interaction effect in both discovery and replication datasets were reported as significant findings. For the significant variants with low allele frequency (MAF $<0.05$ ), we further validated the signals using firth logistic regression, a method designed for rare variants association test to reduce small-sample bias in regular logistic regression (Fig. 1) ${ }^{26}$. The statistical analysis was adjusted for smoking status (ever-and never-smokers) and the first 5 principal components. The analysis was conducted in overall lung cancer as well as adenocarcinoma (No. Cases $=9,791$ ) and squamous (No. Cases $=6,107)$ lung cancer.

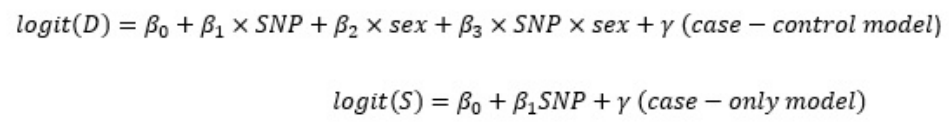

\section{Eqtl Analysis}

Genotype dosage and gene expression rpkm (Reads Per Kilobase Million) data from lung tissue were downloaded from GTEx (phs000424.GTEx.v7.p2). There were 377 individuals with European ancestry available for the analysis, including 244 men and 133 women. Average rpkm for the gene was used if there were duplicated samples. Individuals with rpkm $<0.25$ were removed from the analysis. For imputed variants, we applied a best guess algorithm to assign most likely genotypes to each individual to transform the dosage into genotype. For variants with minor allele frequency (MAF) $>=0.1$, we adopted an additive model: dosage values $<=0.2$ were coded as 0 ; dosage between $0.4-0.6$ were coded as 1 ; and dosage $>=0.8$ was coded as 1 . For variants with MAF $<0.1$, we adopted a dominant model: dosage values $<=0.4$ were coded as 0 (non-carrier group with no risk alleles) and dosage $>=0.6$ was coded as 1 (carrier group with at least 1 risk allele). For each candidate SNP, the boxplot of log2(rpkm) across different genotype were displayed for all the individuals, men and women groups. Student's test was performed to compare the mean log2(rpkm) across different genotype groups and general linear regression was conducted to test SNP-sex interaction in gene expression prediction.

$\log _{2}($ rpkm $) \sim \beta_{0}+\beta_{1} \times S N P+\beta_{2} \times \operatorname{sex}+\beta_{3} \times S N P \times \operatorname{sex}+\gamma($ general linear model $)$

\section{Functional annotation analysis}

Two public functional annotation tools, CADD and RegulomeDB were applied for functional inference. CADD was designed to predict functional variants by integrating diverse information from wide range of function categories ${ }^{16}$. It computed a score inferring the functional ranking of the variants which is helpful for fine mapping. RegulomeDB is a tool designed to predict regulatory DNA elements in the Human genome based on information from GEO, ENCODE and public literature ${ }^{17}$. It adopted SURF (Score of Unified Regulatory Features) model, trained on data from massively parallel reporter assays, to predict the 
functional variants in enhancer and promoter elements. RegulomeDB provides a probability score for the variant being transcription factor binding site (TFBS), promotor or DNase hypersensitivity, etc. PROMO, an online tool to perform computational-based searches for gene expression regulatory sequence motif based on TFBS database, was used to search for the functional motif with sequence containing the candidate variants as input ${ }^{18}$.

Table 3

Functional annotation of candidate SNPs from significant regions.

\begin{tabular}{|c|c|c|c|c|c|}
\hline SNP & rsID & Case-only & CADD & RegulomeDB & \\
\hline POS & SNP & $\mathrm{P}$ & PHRED $^{1}$ & Function Annotation & Probability ${ }^{2}$ \\
\hline 17:49639139:G:A & rs17662871 & $4.29 \times 10^{-8}$ & 2.534 & TF binding or DNase peak & 0.49 \\
\hline 20:15642704:G:A & rs76314075 & $1.33 \times 10^{-7}$ & 1.445 & Other & 0.18 \\
\hline 20:15644218:T:C & rs79942605 & $2.81 \times 10^{-8}$ & 8.228 & TF binding or DNase peak & 0.04 \\
\hline 21:18776825:C:T & rs208900 & $5.87 \times 10^{-6}$ & 0.668 & TF binding or DNase peak & 0.13 \\
\hline 21:18779654:G:A & rs184089 & $5.79 \times 10^{-6}$ & 1.066 & Motif hit & 0.48 \\
\hline 21:18783833:G:A & rs11088636 & $4.24 \times 10^{-6}$ & 0.039 & Other & 0.18 \\
\hline 21:18784296:T:C & rs423598 & $4.78 \times 10^{-6}$ & 2.243 & TF binding or DNase peak & 0.13 \\
\hline 21:18785818:G:A & rs208908 & $4.54 \times 10^{-8}$ & 5.773 & TF binding or DNase peak & 0.92 \\
\hline 21:18787462:T:C & rs208914 & $5.01 \times 10^{-6}$ & 2.944 & TF binding + DNase peak & 0.61 \\
\hline 21:18787572:T:C & rs9637031 & $1.34 \times 10^{-7}$ & 4.018 & TF binding + DNase peak & 0.61 \\
\hline 21:18787948:A:G & rs6517771 & $1.76 \times 10^{-6}$ & 5.095 & Motif hit & 0.34 \\
\hline 21:18788445:A:C & rs1389157 & $1.30 \times 10^{-6}$ & 6.257 & TF binding or DNase peak & 0.03 \\
\hline
\end{tabular}

SNPs with case-only joint $\mathrm{p}$-value $<1 \times 10^{-5}$ were selected for annotation analysis. eQTL displayed the information from GTEx Portal. The annotation information for rs208908 was bolded. 1, scaled CADD score by expressing the rank in order of magnitude terms; 2 , RegulomeDB probability score is ranging from 0 to 1 , with 1 being most likely to be a regulatory variant.

\section{Results}

\section{Novel signals from genome-wide gene-sex interaction analysis}

In the case-only analysis stage, a total of 19,943,415 and 10,359,674 SNPs were tested in the discovery and replication studies, respectively. Figure $2 a$ displays the Manhattan plot of joint case-only analysis of gene-sex interactions in lung cancer. No inflated type I error rate was detected (lambda $=0.98)$. There were 22 SNPs with a joint case-only p-value $<5 \times 10^{-8}$ in meta-analysis and three of them, rs 17662871 , rs 79942605 and rs208908 had case-only p-value $<0.1$ in the replication study. Those SNPs were submitted to case-control interaction analysis using pooled discovery and replication data including 24,223 lung cancer patients and 22,560 controls. All of these SNPs had interaction p-value $<0.05$ in case-control analysis (Supplementary Table S2). All three candidate variants had MAF $<0.05$ and thus were further validated using Firth logistic regression method.

The results from Firth test for the three significant variants are reported in Table 2. SNP rs17662871, located near the CA10 (Carbonic Anhydrase 10) gene, had an OR of 0.69 and case-only p-value of $1.21 \times 10^{-7}$ in discovery study, and OR of 0.80 and p-value of $7.03 \times 10^{-2}$ in the replication study. Further case-control validation using Firth test detected an interaction OR of 0.78 and $p$-value of $4.06 \times 10^{-3}$. SNP rs208908, located near CXADR (Coxsackie Virus And Adenovirus Receptor) gene, had an interaction $\mathrm{p}$-value of $2.84 \times 10^{-6}(\mathrm{OR}=0.71)$ and $4.49 \times 10^{-3}(\mathrm{OR}=0.67)$ in case-only discovery and replication studies, respectively. It has a joint interaction $p$-value of $4.54 \times 10^{-8}(O R=0.70)$ and $p$-value of $1.74 \times 10^{-2}(O R=0.80)$ in case-control analysis. rs79942605 had an $\mathrm{OR}$ of $2.17(\mathrm{p}=$ $\left.2.81 \times 10^{-8}\right)$ in joint case-only analysis. In addition, case-control analysis identified an interaction OR of $1.68\left(\mathrm{p}=1.64 \times 10^{-2}\right)$ in lung cancer cohort. We performed the same test in other lung cancer subt ypes, such as lung adenocarcinoma and squamous lung cancer, etc., but did not identify any significant interactions. 
Table 2

Significant signals identified and replicated in gene-sex interaction analysis in overall lung cancer.

\begin{tabular}{|c|c|c|c|c|c|c|c|c|c|c|c|c|c|}
\hline \multirow[b]{3}{*}{ SNP } & \multirow[b]{3}{*}{ rsID } & \multirow[b]{3}{*}{ MAF } & \multirow[b]{3}{*}{ GENE } & \multicolumn{8}{|c|}{ Case-only } & \multicolumn{2}{|c|}{ Case-control } \\
\hline & & & & \multicolumn{2}{|c|}{ Discovery } & \multicolumn{2}{|c|}{ Replication } & \multicolumn{4}{|c|}{ Meta-analysis } & \multirow[b]{2}{*}{ OR } & \multirow[b]{2}{*}{$P$} \\
\hline & & & & OR & $\mathrm{P}$ & OR & $\mathrm{P}$ & $\mathrm{P}$ & OR & $\mathrm{Q}$ & I & & \\
\hline 17:49639139:G:A & rs17662871 & 0.049 & $\begin{array}{l}\text { CA10 } \\
\text { distal }\end{array}$ & 0.69 & $\begin{array}{l}1.21 \times 10^{-} \\
7\end{array}$ & 0.80 & $\begin{array}{l}7.03 \times 10^{-} \\
2\end{array}$ & $\begin{array}{l}4.29 \times 10^{-} \\
8\end{array}$ & 0.71 & 0.27 & 17.10 & 0.78 & $\begin{array}{l}4.06 \times 10^{-} \\
3\end{array}$ \\
\hline 20:15644218:T:C & rs79942605 & 0.012 & MACROD2 & 2.37 & $\begin{array}{l}9.05 \times 10^{-} \\
8\end{array}$ & 1.67 & $\begin{array}{l}6.34 \times 10^{-} \\
2\end{array}$ & $\begin{array}{l}2.81 \times 10^{-} \\
8\end{array}$ & 2.17 & 0.28 & 14.83 & 1.68 & ${ }_{2}^{1.64 \times 10^{-}}$ \\
\hline 21:18785818:G:A & rs208908 & 0.039 & $\begin{array}{l}\text { CXADR } \\
\text { distal }\end{array}$ & 0.71 & ${ }_{6}^{2.84 \times 10^{-}}$ & 0.67 & $\begin{array}{l}4.49 \times 10^{-} \\
3\end{array}$ & $\begin{array}{l}4.54 \times 10^{-} \\
8\end{array}$ & 0.70 & 0.76 & 0.00 & 0.80 & $\begin{array}{l}1.74 \times 10^{-} \\
2\end{array}$ \\
\hline
\end{tabular}

The results from analysis using Firth logistic regression method were reported. In case-control analysis, all the cases and controls from both discovery and replication study were pooled together for the study. The first 5 principal components and smoking status were adjusted in the analysis. Infor. Q indicated $\mathrm{p}$ values for Cochrane's Q statistic; I indicated I^2 heterogeneity index (0-100); Score indicated the imputation quality score for the variants.

\section{Regional Plot At The Significant Regions}

We examined the regional information around the significant findings. rs17662871, close to $C A 10$ gene, was the single variant achieving genome-wide significance of gene-sex interaction in the region and there were no other variants that were in strong LD with this variant. This variant had an imputation quality score of 0.72 (Fig. 2b Top). SNP rs79942605, located within MACROD2 (Mono-ADP Ribosylhydrolase 2) gene, had an interaction p-value of 2.81x10-8 and OR 2.17 in joint analysis. Another variant rs76314075, in high LD with rs79942605 ( $\left.r^{2}>=0.8\right)$, had an OR of 2.11 and p-value of $1.33 \times 10^{-7}$ in the joint analysis (Fig. 2b Middle). For the third novel variant, rs208908 (OR $\left.=0.70, p=4.54 \times 10^{-8}\right)$, located upstream of CXADR gene, we found 8 variants with joint case-only interaction $\mathrm{p}$-value $<1 \times 10^{-5}$ and the strongly associated variant rs 9637031 had an $\mathrm{OR}$ of 0.70 and $\mathrm{p}$-value of $1.34 \times 10^{-7}$ in the joint analysis (Fig. $2 \mathrm{~b}$ bottom).

\section{Stratified Analysis Of Lung Cancer Risk At Significant Snps}

To explore how sex influenced genetic risk in lung cancer, we conducted stratified lung cancer risk analysis in men and women using discovery and replication combined dataset. We observed very distinct lung cancer risk-patterns between men and women at the three identified variants. For example, rs208908, had a protective effect for lung cancer in women with OR of $0.81\left(p=1.17 \times 10^{-3}\right)$ but had no significant effect in males $(O R=1.03, p=0.59)(F i g .2 c)$. Similarly, SNP rs17662871 had a protective effect for lung cancer in women with OR of $0.86\left(p=1.44 \times 10^{-2}\right)$. We further stratified the analysis by smoking status (ever-. Vs. never-smokers) and the risk effect did not vary by smoking status for these two SNPs. rs79942605, on the other hand, had an increased risk for lung cancer in women $\left(\mathrm{OR}=1.52\right.$, $\mathrm{p}$-value $\left.=4.76 \times 10^{-3}\right)$. And this variant only had risk effect in ever-smoking women $\left(\mathrm{OR}=2.03, \mathrm{p}=4.47 \times 10^{-4}\right)$ while not never-smoking women $\left(\mathrm{OR}=0.60, \mathrm{p}=1.79 \times 10^{-1}\right)$.

\section{Discussion}

Sex is an important biological factor in human disease development and extensive studies have been conducted to demonstrate sex differences in incidence, prognosis, and treatment of various diseases including cancer ${ }^{1-2}$. In 2016, a comprehensive characterization of molecular differences between male and female patients in 13 cancer types using multidimensional genomic data in TCGA (The Cancer Genome Atlas) categorized lung cancer into the strong sex effect group with more extensive sex-biased genetic and molecular signatures ${ }^{29}$. However, differences in risk based on sex are one of the least studied factors in cancer susceptibility. And study of sex disparity in lung cancer risk development is quite limited despite the success of GWAS in lung cancer research during the past decade. A comprehensive gene-sex interaction scanning combined with functional annotation analysis provided exploratory insights about molecular mechanism underlying difference in lung cancer risk between men and women.

Leveraging the rich resource from INTEGRAL-ILCCO lung cancer consortium, we conducted one of the largest gene-sex interaction analysis in overall lung cancer as well as lung cancer subtypes using imputed genotype from 47,000 individuals with European ancestry. By adopting a robust and stringent twostage analysis strategy, we identified three significant gene-sex interactions in overall lung cancer and all the three variants had small minor allele frequency between 0.01 and 0.05 . Considering all the three variants had MAF $<0.05$ and the standard regression method may not preserve the type I error rate for variants with low allele frequency, we further applied the Firth test and validated the signals at the three SNPs. We even retrieved $\sim 200$ SNPs from the three candidate regions and compared the results between regular logistic regression method and Firth logistic regression method. The beta estimation and $p$ values between these two methods were highly concordant with each other implying that our results from case-only analysis were reliable (Supplementary Figure S4). Among the three significant variants, rs17662871 and rs208908 displayed a protective effect for lung cancer in women and no effect in men. And the protective effect did not vary much by smoking status (ever- vs never-) in women. rs79942605, from MACROD2 gene, displayed strong risk effect in only ever-smoking women $\left(\mathrm{OR}=1.52, \mathrm{p}=4.76 \times 10^{-3}\right)$ while no effect in men or never-smoking women was observed. We also conducted the analysis in lung 
adenocarcinoma and squamous lung cancer but didn't identify any significant interactions, possibly due to decreased sample sizes in these subgroups. This suggests that genome-wide scanning for interactions between genes and environmental/biological factors still remains a challenge requiring a more powerful analysis strategy.

eQTL analysis has been very useful in GWAS to provide additional functional evidence for the identified susceptibility loci. However, very few studies were reported about its application in interaction analysis between genes and environmental/biological factors probably because of the limited studies in interaction scanning, restricting the contribution of eQTL in genetic association studies. We explored the application of eQTL in gene-sex interaction study. Take variant rs208908 and CXADR as an example, we didn't identify significant association between rs208908 and CXADR gene in main-effect eQTL analysis $(\mathrm{p}=0.47$, Fig. 3a middle). However, we identified distinct gene expression patterns between men and women and we detected significant gene-sex interaction in $C X A D R$ gene expression prediction $\left(\mathrm{p}=3.85 \times 10^{-3}\right)$. In addition, the signals from gene-sex interactions between lung cancer risk prediction and $C X A D R$ gene expression prediction are highly correlated among the SNPs in high LD with rs208908 ( $r 2>0.8$ ) (Fig. 3a right). The eQTL analysis provided strong evidence suggesting rs208908 is a functional variant with difference in lung cancer risk by sex; and revealed great potential for application of eQTL in large-scale scanning for interactions between genes and environmental/biological factors.

Further functional annotation analysis inferred rs208908 as a transcription factor binding site with probability of 0.92. Based on information from existing TFBS, an 8-bp transcription factor binding site motif containing rs208908 was predicted and the minor allele "A" at rs208908 is predicted to increase the binding of transcription factor of GR- $\beta$ and $Y Y 1$ (Fig. 3b). Both of these transcription factors have been reported to be involved in cancer development and YY1 is a dual function transcription factor and has been implicated as a major driver of many cancers including lung cancer ${ }^{27-28}$. DNase-seq analysis also identified a cis-regulatory region including rs208908 variant in Human cells. These results, combined with eQTL analysis, suggested rs208908 regulated CXADR gene expression by interacting with cellular factors such as transcription factors that function through recognition of conserved sequence motif located upstream of gene coding sequence. In 2018, a large-scale GWAS including 370,000 individuals from European population showed that CXADR gene was associated with lung function (FEV1/FVC ratio) ${ }^{30}$. These multiple lines of evidence, from the results from our integrated study to that from previous reports, suggested a disease model for SNP rs208908 with sexual disparity in lung cancer risk. SNP rs208908 has different MAF between women (0.030) and men (0.025). This difference combined with differentiated gene expression regulation mechanism between men and women, leads to different gene expression patterns between these groups resulting in different risk between male and female groups (Fig. 3c). In summary, rs208908 affects lung cancer risk differentially in men and women through downstream CXADR gene expression regulation in lung tissues.

For the other two variants, rs17662871 (close to CA10) and rs79942605 (within MACROD2), very few supporting variants were identified nearby, which is not unusual for variants with low allele frequency. The low allele frequency also makes eQTL analysis challenging. rs79942605 was not available in GTEx and there were only a few samples with homogenous minor-allele genotype at rs17662871 in GTEx genotype data, which limited our ability to investigate the gene expression pattern across different genotype groups. Both $C A 10$ and MACROD2 were reported to be associated with smoking behavior and age at menarche in Caucasian ${ }^{31-32}$. Controversial results have been reported for association between age at menarche and lung cancer risk ${ }^{33-35}$. Our study detected an effect for lung cancer in only women group in these two regions, especially rs79942650 from MACROD2 gene which has a risk effect in only smoking women, suggesting that sex plays an important role in lung cancer susceptibility and may interact with smoking behavior in cancer risk.

In summary, we conducted a large-scale gene-sex interaction scanning in lung cancer and we identified three significant variants with different risk effects based on sex. Our study is one of the first studies to examine sex disparity in lung cancer development and our results provided insights about the genetic and molecular mechanism underlying the differences in lung cancer susceptibility between men and women. Our findings also suggested quite a few variants still remain undetected in lung cancer, including those with low allele frequency requiring a very large sample size. Variants affecting disease risk through interaction with environmental and biological factors require a more robust analysis strategy.

\section{Declarations}

\section{Acknowledgment}

The Genotype-Tissue Expression (GTEx) Project was supported by the Common Fund of the Office of the Director of the National Institutes of Health, and by $\mathrm{NCl}, \mathrm{NHGRI}, \mathrm{NHLBI}, \mathrm{NIDA}, \mathrm{NIMH}$, and NINDS. The data used for the analyses described in this manuscript were obtained from: dbGaP accession number phs000424.v7.p2.

We thank the grants U19-CA203654 (NIH), RR170048 (Cancer Prevention Research Institute of Texas (CPRIT)), RR160097T (CPRIT), RR180061 (CPRIT), U01 CA243483-02 (Genetic Epidemiology of Lung Cancer Consortium (GELCC)), X01HG007491, Department of Health and Human Services contracts HHSN26820100007C, HHSN268201700012C, 75N92020C00001, and 1R21CA235464-01A1 (NIH) for support. CARET is funded by the National Cancer Institute, National Institutes of Health through grants U01-CA063673, UM1-CA167462, and U01-CA167462. The Harvard Lung Cancer Study is funded by NIH (NCl) grant U01CA209414 (Boston Lung Cancer Survival Cohort). The Liverpool Lung Project is supported by MPAD. Roy Castle Lung Foundation (UK). The EAGLE study was supported by the intramural program of the Division of Cancer Epidemiology and Genetics, National Cancer Institute, NIH. Joan E. The ReSoLuCENT study is funded by the Weston Park Hospital Cancer Charity. Acknowledgement to the George Isaac Family Fund for Cancer Research for grant support. Bailey-Wilson was supported by the Intramural Program of the National Human Genome Research Institute, NIH. Chris Amos and Chao Cheng are Research Scholars of the Cancer Prevention Institute of Texas.

\section{Author contribution}

Yafang Li designed and planned the study, presented the results and wrote the manuscript. Xiangjun Xiao assisted the data preparation and genome-wide gene-sex interaction analysis; Jianrong Li and Chao Cheng assisted the motif analysis in functional annotation; Jinyoung Byun assisted the data preparation; 
Yohan Bossé assisted the eQTL analysis. Christopher I. Amos conceived and supervised the study. The other authors contributed to data collection. All authors discussed the results and commented on the manuscript.

\section{Materials \& Correspondence}

Correspondence to Yafang Li or Christopher I. Amos. Material requests should be addressed to Chris Amos.

\section{Competing interests}

The authors declare no competing interests.

\section{References}

1. Zhang, E.A. \& Wynder, E.L. Differences in lung cancer risk between men and women: examination of the evidence. J Nat/ Cancer Inst 88(3-4), 183-92. doi: 10.1093/jnci/88.3-4.183 (1996).

2. Kabir, Z., Connolly, G.N., Clancy, L. Sex-differences in lung cancer cell-types? An epidemiologic study in Ireland. U/ster Med J 77(1), 31-35 (2008).

3. Haenszel, W. \& Taeuber, K.E. Lung cancer mortality as related to residence and smoking histories. II. White females. J Nat/ Cancer Inst 32, 803-838 (1964).

4. Hammond, E.C. Smoking in relation to the death rates of one million men and women. Natl Cancer Inst Monogr 19, 127-204 (1966).

5. Doll, R., Gray, R., Hafner, B. \& Peto, R. Mortality in relation to smoking: 22 years' observations on female British doctors. Br Med J 280, 967-971 (1980).

6. Jemal, A., et al. Higher Lung Cancer Incidence in Young Women Than Young Men in the United States. N Engl J Med 378, $1999-2009$. 10.1056/NEJMoa1715907 (2018).

7. Lan, Q., et al. Genome-wide association analysis identifies new lung cancer susceptibility loci in never-smoking women in Asia. Nat Genet 44(12), 13301335 (2012).

8. Wang, Z., et al. Meta-analysis of genome-wide association studies identifies multiple lung cancer susceptibility loci in never-smoking Asian women. Hum Mol Genet 25:3(1), 620-629 (2016).

9. Murcray, C.E., Lewinger, J.P. \& Gauderman W.J. Gene-environment interaction in genome-wide association studies. Am J Epidemio/ 169, 219-226 (2009).

10. Li, Y., et al. Genome-wide interaction study of smoking behavior and non-small cell lung cancer risk in Caucasian population. Carcinogenesis 39(3), 336346 (2018).

11. Edwards, S.L., Beesley, J., French, J.D. \& Dunning, A.M. Beyond GWASs: illuminating the dark road from association to function. Am J Hum Genet 93 , 779-97. doi: 10.1016/j.ajhg.2013.10.012 (2013).

12. Hrdlickova, B., de Almeida, R.C., Borek, Z. \& Withoff S. Genetic variation in the non-coding genome: Involvement of micro-RNAs and long non-coding RNAs in disease. Biochim Biophys Acta 1842, 1910-22. doi: 10.1016/j.bbadis.2014.03.011 (2014).

13. Hazelett, D.J., et al. Comprehensive functional annotation of 77 prostate cancer risk loci. PLoS Genet 10, e1004102 (2014).

14. Pomerantz, M. M. et al. The 8q24 cancer risk variant rs6983267 shows long-range interaction with MYC in colorectal cancer. Nat Genet $41,882-884$ (2009).

15. Smemo, S. et al. Obesity-associated variants within FTO form long-range functional connections with IRX3. Nature 507, 371-375 (2014).

16. Rentzsch, P., Witten, D., Cooper, G.M., Shendure, J. \& Kircher, M. CADD: predicting the deleteriousness of variants throughout the human genome. Nucleic Acids Res 47(D1), D886-D894. doi: 10.1093/nar/gky1016 (2019).

17. Boyle, A.P., et al. Annotation of functional variation in personal genomes using RegulomeDB. Genome Research 22(9), 1790-1797. PMID: 22955989 (2012).

18. Messeguer, X., Escudero, R., Farré, D., Nuñez, O., Martínez, J. \& Albà, M. PROMO: detection of known transcription regulatory elements using speciestailored searches. Bioinformatics 18:2, 333-334 (2002).

19. Byun, J., et al. Trans-ethnic genome-wide meta-analysis of 35,732 cases and 34,424 controls identifies novel genomic cross-ancestry loci contributing to lung cancer susceptibility. Preprint at https://doi.org/10.1101/2020.10.06.20207753 (2020).

20. McKay, J.D. et al. Large-scale association analysis identifies new lung cancer susceptibility loci and heterogeneity in genetic susceptibility across histological subtypes. Nat Genet 49:1126-1132 (2017).

21. Amos, C.I., et al. The OncoArray Consortium: A Network for Understanding the Genetic Architecture of Common Cancers. Cancer Epidemiol Biomarkers Prev 26,126-135 (2017).

22. Kachuri, L., et al. Fine mapping of chromosome 5 p15.33 based on a targeted deep sequencing and high-density genotyping identifies novel lung cancer susceptibility loci. Carcinogenesis 37, 96-105 (2016).

23. Landi, M.T. et al. Environment and Genetics in Lung cancer Etiology (EAGLE) study: an integrative population-based case-control study of lung cancer. BMC Public Health 8, 203 (2008).

24. Ji, X., et al. Identification of susceptibility pathways for the role of chromosome 15q25.1 in modifying lung cancer risk. Nat Commun 9, 3221 (2018).

25. Mitchell, K.A., et al. Relationship between West African ancestry with lung cancer risk and survival in African Americans. Cancer Causes Contro/ 30, 12591268 (2019)

26. Wang X. Firth logistic regression for rare variant association tests. Front Genet 5,187 (2014). 
27. Sarvagalla, S., Kolapalli, S.P. \& Vallabhapurapu, S. The Two Sides of YY1 in Cancer: A Friend and a Foe. Front Onco/ 20

https://doi.org/10.3389/fonc.2019.01230 (2019).

28. McBeth, L., et al. Glucocorticoid receptor beta increases migration of human bladder cancer cells. Oncotarget 7(19), 27313-27324 (2016).

29. Yuan, Y. Comprehensive characterization of molecular differences in cancer between male and female patients. Cancer Cel/ 29, 711-722 (2016).

30. Kichaev, G., et al. Leveraging polygenic functional enrichment to improve GWAS power. Am J Hum Genet 104(1), 65-75. doi:10.1016/j.ajhg.2018.11.008 (2019).

31. Brazel, D.M., et al. Exome chip meta-analysis fine maps causal variants and elucidates the genetic architecture of rare coding variants in smoking and alcohol use. Biol Psychiatry 85(11), 946-955 (2019).

32. Gill, D., et al. Age at menarche and lung function: a Mendelian randomization study. Eur J Epidemio/32(8), 701-710 (2017).

33. Magnus, M.C., et al. Identifying potential causal effects of age at menarche: a Mendelian randomization phenome-wide association study. $B M C$ Medicine 18(71) (2020).

34. He, F., et al. The relationship of lung cancer with menstrual and reproductive factors may be influenced by passive smoking, cooking oil fumes, and tea intake. Medicine (Baltimore) 96(46), e8816 (2017).

35. Khedher, S.B., et al. Menstrual and reproductive factors and lung cancer risk: A pooled analysis from the international lung cancer consortium. Int $J$ Cancer 141(2), 309-323. doi: 10.1002/ijc.30750 (2017).

\section{Figures}

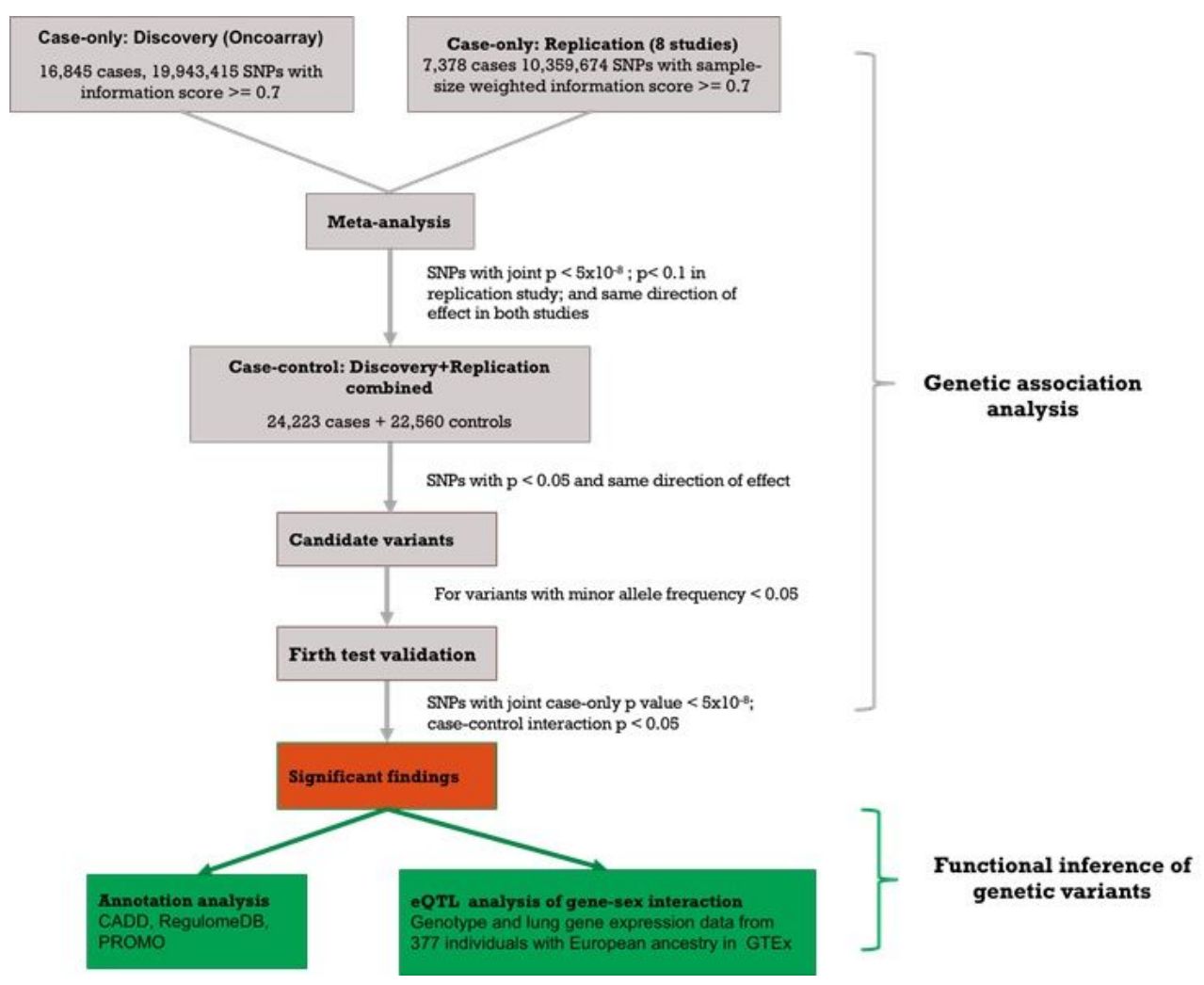

Figure 1

Flow chart of analysis strategy in genome-wide gene-sex interaction analysis in lung cancer. In genetic association analysis, all the tested SNPs have information score $>=0.7$ in discovery data; information score $>0.2$ in each of the 8 studies and sample-size weighted information score $>=0.7$ in replication data. Smoking status (ever-vs never-smokers) and first 5 principal components were adjusted in case-only, case-control and firth validation analysis. 


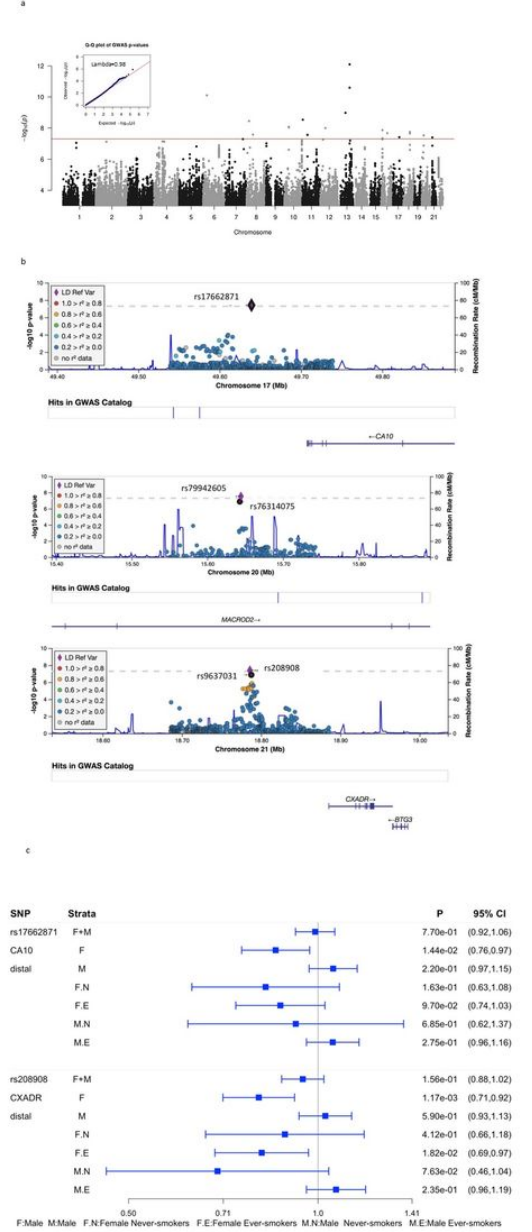

\section{Figure 2}

Plot of signals from genome-wide gene-sex interaction analysis in lung cancer. a, Manhattan plot of p-values from case-only meta-analysis. No inflated type I error was detected in the analysis (lambda=0.98). b, regional plot at three significant regions. c, forest plot of stratified lung cancer risk for the most significant SNP from each region. 

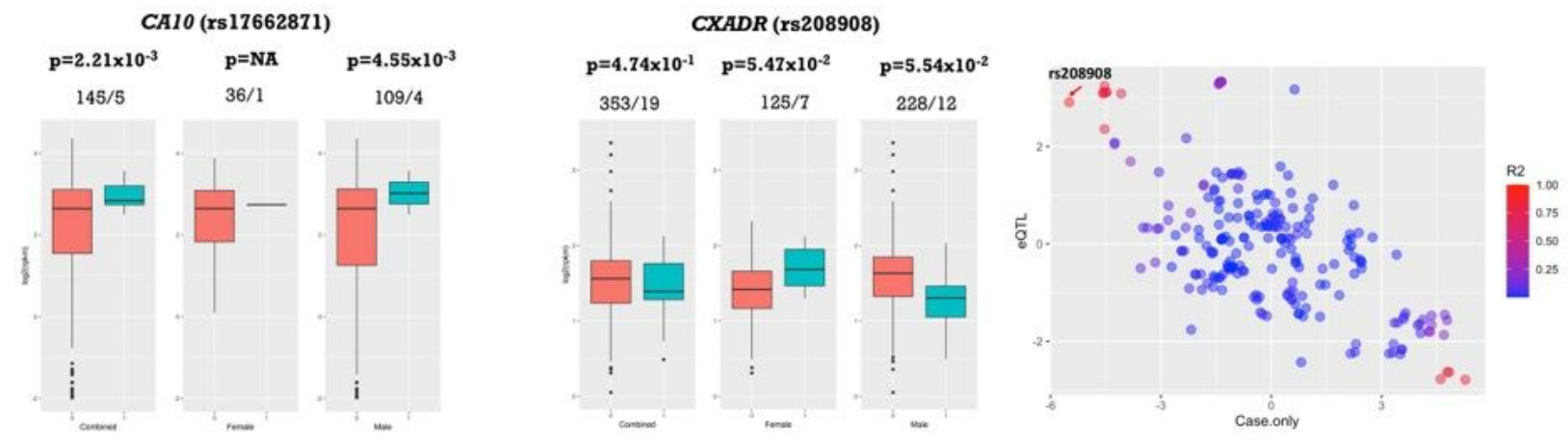

b

c

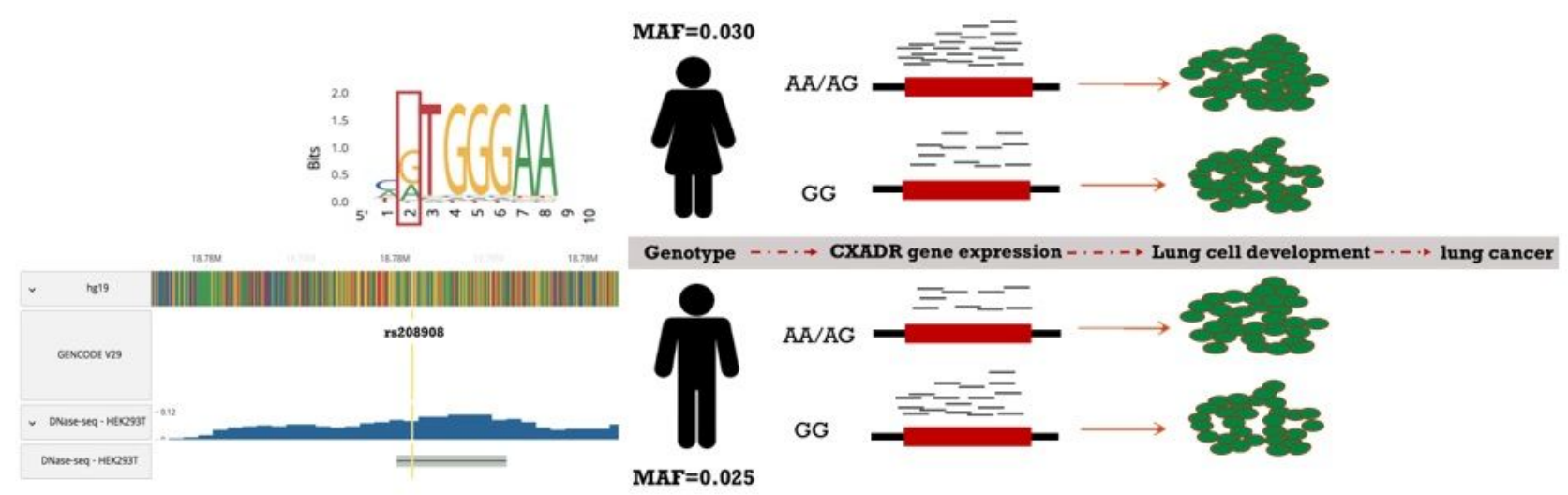

Figure 3

Functional analysis of identified variants. a, eQTL analysis at candidate variants. Left and middle, box plot of gene expression from lung between individuals with none (0) and at least on risk allele (1) in female and male combined, female and male groups for CA10 and CXADR, respectively. The data from samples with Caucasian ancestry in GTEx were used in the analysis. Individuals with $\mathrm{rpkm}<0.25$ were removed from the analysis. P-values and number of samples were labeled above each plot. rs208908 was genotyped in GTEx; rs17662871 was and imputed SNP and SNP dosage <=0.4 was coded as 0 ; SNP dosage $>=0.6$ was coded as 1 . Linear regression analysis of gene-sex interaction in predicting CXADR gene expression displayed $p=3.85 \times 10-3$. Right, Scatter plot of $z-$ scores from 229 SNPs, ranging from 18745702 to 18806105 (hg19) on chromosome 21, were displayed on the plot. X-axis represented gene-sex interaction analysis in lung cancer risk; and Y-axis represented the z-scores from gene-sex interaction analysis in CXADR gene expression prediction. LD r2 was computed for each of the SNPs with rs208908 as reference. b, Functional inference of rs208908. The predicted binding motif at rs208908 (highlighted in red color) for transcription factor (TF) using PROMO (Upper). DNase-seq analysis in HEK293T (Human embryonic kidney) cells detected a peak covering rs208908 suggesting it was located within a cis-regulatory DNA sequence element (RegulomeDB bottom). c, Presumptive disease risk model at rs208908. This variant, located upstream of CXADR, may affect lung cancer risk through gene expression regulation.

\section{Supplementary Files}

This is a list of supplementary files associated with this preprint. Click to download.

- GenesexmanuscriptAppendix06162021.docx 